\section{(2) OPEN ACCESS}

\title{
Incidence, predictors, and prognostic impact of recurrent acute myocardial infarction in China
}

\author{
Jiali Song, ${ }^{1}$ Karthik Murugiah ${ }^{2,3}$ Shuang Hu, ${ }^{1}$ Yan Gao, ${ }^{1}$ Xi Li, ${ }^{1}$ Harlan M Krumholz, ${ }^{2,3,4}$ \\ Xin Zheng (1) , ${ }^{1}$ for the China PEACE Collabortive Group
}

- Additional material is published online only. To view please visit the journal online (http://dx.doi.org/10.1136/ heartjnl-2020-317165).

For numbered affiliations see end of article.

\section{Correspondence to} Dr. Xin Zheng, National Clinical Research Center for Cardiovascular Diseases, NHC Key Laboratory of Clinical Research for Cardiovascular Medications, State Key Laboratory of Cardiovascular Disease, Fuwai Hospital, Chinese Academy of Medical Sciences and Peking Union Medical College, National Center for Cardiovascular Diseases, Beijing China; xin.zheng@fwoxford.org

JS and $\mathrm{KM}$ are joint first authors. HMK and XZ are joint senior authors.

Received 22 April 2020 Revised 1 August 2020 Accepted 5 August 2020 Published Online First 16 September 2020

\section{ABSTRACT}

Background Incidence, predictors, and prognostic impact of recurrent acute myocardial infarction (AMI) after initial AMI remain poorly understood. Data on recurrent $A M I$ in China is unknown.

Methods Using the China Patient-centred Evaluative Assessment of Cardiac Events (PEACE)-Prospective AMI Study, we studied 3387 patients admitted to 53 hospitals for AMI and discharged alive. The association of recurrent $A M I$ with 1-year mortality was evaluated using time-dependent Cox regression. Recurrent AMI events were classified as early (1-30 days), late (31-180 days), and very late (181-365 days). Their impacts on 1-year mortality were estimated by Kaplan-Meier methodology and compared by the log-rank test. Multivariable modelling was used to identify factors associated with recurrent AMI.

Results The mean (SD) age was 60.7 (11.9) years and $783(23.1 \%)$ were women. The observed 1-year recurrent $\mathrm{AMI}$ rate was $2.5 \%(95 \% \mathrm{Cl} 2.00$ to 3.07$)$ with $35.7 \%$ events occurring within the first 30 days. Recurrent AMI was associated with 1-year mortality with an adjusted HR of 25.42 (95\% Cl 15.27 to 42.34). Early recurrent $\mathrm{AMI}$ was associated with the highest 1-year mortality rate of $53.3 \%$ (log-rank $p<0.001)$. Predictors of recurrent AMI included age 75-84, in-hospital percutaneous coronary intervention, heart rate $>90 \mathrm{~min} /$ beats at initial admission, renal dysfunction, and not being prescribed any of guideline-based medications at discharge.

Conclusions One-third of recurrent AMI events occurred early. Recurrent AMI is strongly associated with 1-year mortality, particularly if early. Heightened surveillance during this early period and improving prescription of recommended discharge medications may reduce recurrent $A M I$ in China.

\section{INTRODUCTION}

Patients who survive acute myocardial infarction (AMI) in the acute phase remain at risk for recurrent AMI after discharge. Although recurrent AMI rates have declined over time, ${ }^{1-4}$ mortality rates after recurrent AMI remain high, with one-third of patients dying within 1 year after a recurrent AMI. ${ }^{2}$ Comprehensively characterising the timing, predictors, and prognostic effect of recurrent AMI can help develop effective strategies to prevent these events of consequence and improve overall survival rates of patients.

Prior studies using data from clinical trials to assess the predictors and prognostic impact of recurrent AMI have yielded valuable insights on this topic. ${ }^{5-9}$ However, these results may not generalisable to real-world populations. On the other hand, studies using administrative claims ${ }^{1-4}$ are more representative of the population providing reliable incidence estimates, but unable to assess predictors of recurrent AMI in detail. Further, although previous studies highlight that a large proportion of recurrent AMI events occur relatively soon within 30 days of hospital discharge from initial AMI, ${ }^{10} 11$ we lack a deeper understanding of the extent to which the risk for these events change with time beyond the 30-day period.

Studying recurrent AMI is especially important for countries such as China which are facing an epidemic of cardiovascular disease. By 2030, the number of individuals with AMI in China is expected to reach 23 million, ${ }^{12}$ which translates to a large population vulnerable to recurrent AMI events. Developing a deeper understanding of the factors associated with recurrent AMI, the period of heightened vulnerability, and the extent to which recurrent AMI and the timing of recurrent $\mathrm{AMI}$ impacts postdischarge mortality in China can help devise strategies to reduce the burden of recurrent AMI.

Accordingly, in this study we use data from the China Patient-centered Evaluative Assessment of Cardiac Events (PEACE)-Prospective AMI Study ${ }^{13}$ to evaluate the timing and frequency of recurrent AMI, the association between recurrent AMI and 1-year mortality, and the prognostic impact of the timing of recurrent AMI on mortality. We then study predictors of recurrent AMI eventsincluding patient characteristics and treatments at discharge.

\section{METHODS}

\section{Study design and study population}

The China PEACE-Prospective AMI Study has been previously described in detail. ${ }^{13}$ In brief, it is a large prospective cohort study that consecutively recruited patients from 53 hospitals (35 tertiary and 18 secondary hospitals) located in 21 of 31 provinces in China between December 2012 and May 2014. Patients were eligible if they were aged 18 years or older and hospitalised for AMI within 24 hours of onset of symptoms. The diagnosis of AMI was consistent with the third universal definition. ${ }^{14}$ Among the 3447 patients who agreed to be enrolled in this study and followed prospectively, we excluded patients who were transferred to another
To cite: Song J, Murugiah K, Hu, et al. Heart 
facility $(n=13)$, died prior to discharge or were withdrawn from treatment during hospitalisation $(\mathrm{n}=19)$, were lost to follow-up at 1 year after discharge $(n=25)$, or had conflicting or missing information on the days to last follow-up $(n=3)$. Finally, 3387 $(99.2 \%)$ eligible patients were included in this study.

All patients provided written informed consent. The study is registered at www.clinicaltrials.gov (NCT01624909).

\section{Patient and public involvement}

Patients and/or the public were not involved in the design, conduct, reporting or dissemination plans of this research.

\section{Data collection and variable definitions}

Sociodemographic and clinical characteristics, as well as treatments and outcomes were obtained by central medical record abstraction and were supplemented with information from patient interviews. Patients completed a baseline interview during the index hospitalisation for AMI and were followed up at 1,6 , and 12 months following discharge. Site investigators completed the electronic questionnaires on a tablet computer during the interview, which allowed real-time check to ensure the accuracy and completeness of data. Copies of medical records of all hospitalisations during follow-up were collected for outcome event adjudication. Additionally, the abstraction quality was monitored by random auditing of $5 \%$ of the medical records, with overall variable accuracy exceeding 98\%.

Patient characteristics included demographics, socioeconomic factors, cardiac history, cardiovascular risk factors, comorbidities, time between onset of symptoms and admission, prehospital emergency medical services, in-hospital diagnoses, vital signs at admission, diagnostic tests, in-hospital treatments and discharge medications, and in-hospital complications. Renal dysfunction was defined as blood urea nitrogen $>40 \mathrm{mg} / \mathrm{dL}$ or creatinine $>2.5 \mathrm{mg} / \mathrm{dL}$.

To assess the baseline risk during the index AMI hospitalisation, we calculated a risk score for 1-year all-cause mortality for each patient. The risk score was based on a risk model that was reported in a previous study. ${ }^{15}$ We stratified patients into three risk groups based on the distribution of the baseline risk scores for 1-year mortality: low (<10th percentile), average (10th-90th percentile), and high ( $>90$ th percentile).

We also evaluated the prescription rate for the following five guideline-based therapies at discharge consistent with American Heart Association/American College of Cardiology (AHA/ ACC) performance measures for patients with AMI, including aspirin, beta-blocker, statin, clopidogrel, and angiotensinconvertingenzyme inhibitor/angiotensin receptor blocker. ${ }^{16}$ Prescription rates were calculated among patients considered ideal candidates for each treatment. A composite prescription rate was calculated by determining the number of these therapies for which each patient was ideal (the denominator) and then determining the number of those treatments that the patient received (the numerator). So, this can be understood as the percentage of opportunities for secondary prevention treatments that were fulfilled.

The outcomes in our study were death from any cause, cardiac death, and recurrent AMI within 1 year using the index AMI discharge as the time zero. Information on all clinical events including recurrent AMI and death was systematically obtained from relevant hospital records and death certificates collected during follow-up interview by local clinic staff. If a patient was unable or unwilling to attend the scheduled interview, information was collected over the telephone through a direct conversation between study physicians and patients or their relatives. All events were centrally adjudicated by trained cardiologists at the national coordinating center, according to standard approaches used in international large-scale trials. ${ }^{17}$

\section{Statistical analysis}

Continuous variables were reported as median with interquartile range (IQR) or mean with standard deviation (SD) and compared with the Kruskal-Wallis test. Categorical variables were presented as percentages and compared with the $\chi^{2}$ test. Differences in demographic and clinical characteristics of patients with and without recurrent AMI during 1-year follow-up were examined. To investigate the association between the recurrent AMI and 1-year mortality, we fitted a frailty Cox model with hospitalspecific random effect that modelled 1-year death as a function of the first recurrent AMI and adjusted for baseline mortality risk score. Recurrent AMI was modelled as a time-dependent variable in which the variable represents information on the occurrence of an event (yes/no) and the time of the occurrence. We also conducted an additional analysis with the last recurrent event as the time-dependent variable in the Cox model. To evaluate the impact of timing of the first recurrent AMI on the risk of 1-year mortality, we categorised recurrent AMI into three subgroups based on the number of days to recurrent AMI after discharge: early (1 to 30 days), late (31 to 180 days), and very late (181 to 365 days). Their impacts on mortality within 1 year were modelled using Kaplan-Meier methods and compared using the log-rank test. We also assessed the impact of the timing of recurrent AMI on 1-year cardiac death for sensitivity analysis. To examine the predictors of recurrent AMI, we fitted a frailty Cox regression with hospital-specific random effect to model recurrent AMI as a function of patient characteristics and discharge medications [a binary variable indicated whether any of eligible guideline-based medications being prescribed or not for an individual patient $(1=$ not being prescribed any of guideline-based medications; $0=$ being prescribed at least one guideline-based medications)]. For patients experiencing more than one recurrent AMI events, the first recurrent AMI event was counted. The proportional hazard assumption was assessed by including predictors-by-time interactions in the model and testing their statistical significance. No interaction terms reached statistical significance indicating that the proportional hazard assumption was satisfied.

Missing covariate data were imputed using the multiple imputation method with 10 imputations. All analyses were performed on the data with missing value replaced by the average of the 10 imputations. ${ }^{18}$ The actual missing rates ranged from $0.2 \%$ (systolic blood pressure at admission) to $2.7 \%$ (blood glucose) (Online Supplemental Table S2). Analyses were conducted using SAS V.9.4, 64-bit Windows (SAS Institute, Cary, North Carolina, USA). All tests of significance were two-tailed, with a level of significance set at an alpha of 0.05 .

\section{RESULTS}

\section{Baseline characteristics}

Our study sample included 3387 patients with a mean (SD) age as 60.7 (11.9) years, $783(23.1 \%)$ of which were women. Compared with patients without recurrent AMI, patients who experienced recurrent AMI were older, more commonly female, had a higher proportion of hypertension, diabetes mellitus, renal dysfunction, previous AMI, previous heart failure, and previous angina. They were more likely to 
Table 1 Baseline characteristics of patients with and without recurrent AMI after initial AMI

\begin{tabular}{|c|c|c|c|c|}
\hline & All patients & Recurrent AMI & No recurrent AMI & $P$ value \\
\hline & $\mathrm{N}=3387$ & $\mathrm{n}=84$ & $n=3303$ & \\
\hline Sociodemographics & & & & \\
\hline Age, mean (SD) & $60.7(11.9)$ & $66.2(12.8)$ & $60.5(11.8)$ & $<0.001$ \\
\hline Age category, years, n (\%) & & & & $<0.001$ \\
\hline $18-44$ & $307(9.1)$ & $5(6.0)$ & $302(9.1)$ & \\
\hline $45-64$ & $1774(52.4)$ & $26(31.0)$ & $1748(52.9)$ & \\
\hline $65-74$ & $861(25.4)$ & $29(34.5)$ & $832(25.2)$ & \\
\hline $75-84$ & $407(12.0)$ & $24(28.6)$ & $383(11.6)$ & \\
\hline$\geq 85$ & $38(1.1)$ & $0(0.0)$ & $38(1.2)$ & \\
\hline Female, n (\%) & $783(23.1)$ & $28(33.3)$ & $755(22.9)$ & 0.025 \\
\hline College degree, $\mathrm{n}(\%)$ & $462(13.6)$ & $9(10.7)$ & $453(13.7)$ & 0.429 \\
\hline Employed, n (\%) & 1420 (41.9) & $20(23.8)$ & $1400(42.4)$ & $<0.001$ \\
\hline Medical history and comorbidities, n (\%) & & & & \\
\hline History of angina & $134(4.0)$ & $7(8.3)$ & $127(3.8)$ & 0.037 \\
\hline History of acute myocardial infarction & $267(7.9)$ & $12(14.3)$ & $255(7.7)$ & 0.027 \\
\hline History of heart failure & $787(23.2)$ & $33(39.3)$ & $754(22.8)$ & $<0.001$ \\
\hline Dyslipidaemia & $1885(55.7)$ & $58(69.0)$ & $1827(55.3)$ & 0.012 \\
\hline Hypertension & $230(6.8)$ & $12(14.3)$ & $218(6.6)$ & 0.006 \\
\hline Diabetes & $1005(29.7)$ & $36(42.9)$ & $969(29.3)$ & 0.007 \\
\hline Renal dysfunction & $694(20.5)$ & $33(39.3)$ & $661(20.0)$ & $<0.001$ \\
\hline Clinical characteristics, n (\%) & & & & \\
\hline Systolic blood pressure at admission $<100 \mathrm{~mm} \mathrm{Hg}$ & $264(7.8)$ & $6(7.1)$ & $258(7.8)$ & 0.822 \\
\hline Heart rate at admission $>90$ beats $/ \mathrm{min}$ & $450(13.3)$ & $21(25.0)$ & $429(13.0)$ & 0.001 \\
\hline Ejection fraction $<40 \%$ & $245(7.2)$ & $8(9.5)$ & $237(7.2)$ & 0.412 \\
\hline ST-segment elevation AMI & $2768(81.7)$ & $61(72.6)$ & $2707(82.0)$ & 0.029 \\
\hline Reperfusion therapies, n (\%) & & & & \\
\hline $\mathrm{PCl}$ & $2363(69.8)$ & $33(39.3)$ & $2330(70.5)$ & $<0.001$ \\
\hline Thrombolytic therapy & 489 (14.4) & $11(13.1)$ & 478 (14.5) & 0.723 \\
\hline In-hospital medications, n (\%) & & & & \\
\hline Aspirin & $3085(91.1)$ & 79 (94.0) & $3006(91.0)$ & 0.334 \\
\hline Clopidogrel & $3231(95.4)$ & $74(88.1)$ & 3157 (95.6) & 0.001 \\
\hline Beta-blocker & $2759(81.5)$ & $67(79.8)$ & $2692(81.5)$ & 0.685 \\
\hline ACEI/ARB & $2486(73.4)$ & $54(64.3)$ & $2432(73.6)$ & 0.056 \\
\hline Statin & 3337 (98.5) & $80(95.2)$ & 3257 (98.6) & 0.012 \\
\hline Discharge medications, $\mathrm{n}(\%)$ & & & & \\
\hline Aspirin & 3187 (94.1) & $73(86.9)$ & $3114(94.3)$ & 0.005 \\
\hline Clopidogrel & $3088(91.2)$ & $70(83.3)$ & 3018 (91.4) & 0.01 \\
\hline Beta-blocker & $2376(70.2)$ & $60(71.4)$ & $2316(70.1)$ & 0.796 \\
\hline ACEI/ARB & $2136(63.1)$ & $52(61.9)$ & $2084(63.1)$ & 0.824 \\
\hline Statin & $3193(94.3)$ & $74(88.1)$ & $3119(94.4)$ & 0.014 \\
\hline Length of stay, median (IQR) & 11 (8 to 14$)$ & 12 (8 to 14.5$)$ & 11 (8 to 14$)$ & 0.286 \\
\hline
\end{tabular}

$\mathrm{ACEl}$, angiotensin-converting enzyme inhibitor; $\mathrm{AMI}$, acute myocardial infarction; $\mathrm{ARB}$, angiotensin receptor blocker; $\mathrm{CABG}$, coronary artery bypass grafting; $\mathrm{PCl}$, percutaneous coronary intervention.

have a higher heart rate at admission and were less likely to have an ST-segment elevation AMI. In terms of in-hospital treatments during the primary event, patients with recurrent AMI were less likely to receive percutaneous coronary intervention (PCI), clopidogrel, and statin. At discharge, patients with recurrent AMI were less likely to receive aspirin, clopidogrel, and statin $(\mathrm{p}<0.05)$ (Table 1$)$.

\section{Frequency and timing of recurrent AMI}

Median time to first recurrent AMI was 79 (IQR 4-180) days after discharge. For patients who did not receive all treatments for which they were considered ideal, the median time to first recurrent AMI was 42 (IQR 3-177) days after discharge. There were 84 [2.5\% (95\% CI 2.00 to 3.07)] patients having a total of 90 recurrent AMI events after initial AMI during the 1-year follow-up. Of these 84 patients, 30 (35.7\%), 33 (39.3\%), and 21 (25.0\%) experienced recurrent AMI events that were in the early ( 1 to 30 days), late (31 days to 180 days), and very late (181 days to 365 days) period, respectively. (Figure 1) The observed 1-year recurrent AMI rate among patients aged $\geq 65$ years was $4.1 \%$ (95\% CI 3.04 to 5.21). The proportion of high-risk patients among patients having early recurrent AMI was higher than that among patients having late and very late recurrent AMI $(46.7 \%$ vs $22.2 \%, \mathrm{p}=0.02)$. Five $(6.0 \%)$ patients had more than one recurrent AMI event. The median duration between the first and the last recurrent AMI event was 59 days (IQR 21-106) (Online Supplemental Table S1). 


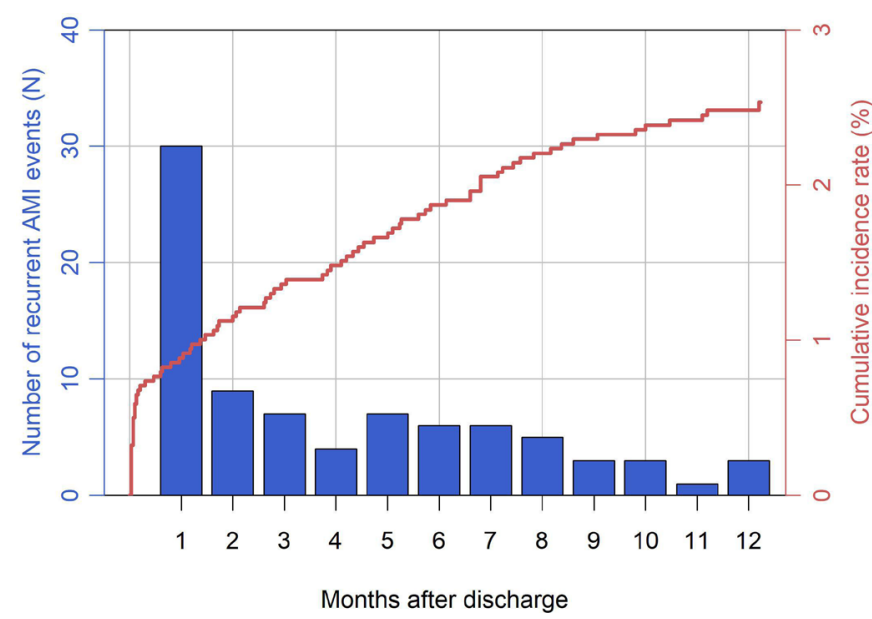

Figure 1 Distribution of first recurrent acute myocardial infarction (AMI) after discharge from initial AMI.

\section{Association between recurrent AMI and 1-year mortality}

The observed 1-year mortality rate in the study cohort was $2.8 \%$ (95\% CI 2.28 to 3.41). Among these 95 patients who died in 1-year follow-up, 27 (28.4\%) had experienced recurrent AMI. Among 84 patients experiencing recurrent AMI, 27 (32.1\%) died during the 1-year follow-up. The median time from first recurrent AMI to death was 1 (IQR 1-25) days. After adjustment for baseline mortality risk score, recurrent AMI was strongly associated with 1-year mortality with an adjusted HR of 25.42 (95\% CI 15.27 to 42.34 ) and 26.20 (95\% CI 15.69 to 43.77) using the first event and the last event as a timedependent variable, respectively (Online Supplemental Figure S1).

Figure 2 depicts time to death in 1 year following discharge, according to the timing of recurrent AMI. Early recurrent AMI conferred the highest 1-year mortality rate of $53.3 \%$ (logrank $\mathrm{p}<0.001)$. The sensitivity analysis yielded similar results regarding the association of the timing of recurrent AMI with 1-year cardiac death (Online Supplemental Figure S2). Among five patients with more than one recurrent AMI events, 4 $(80.0 \%)$ patients died within 1 year after discharge.

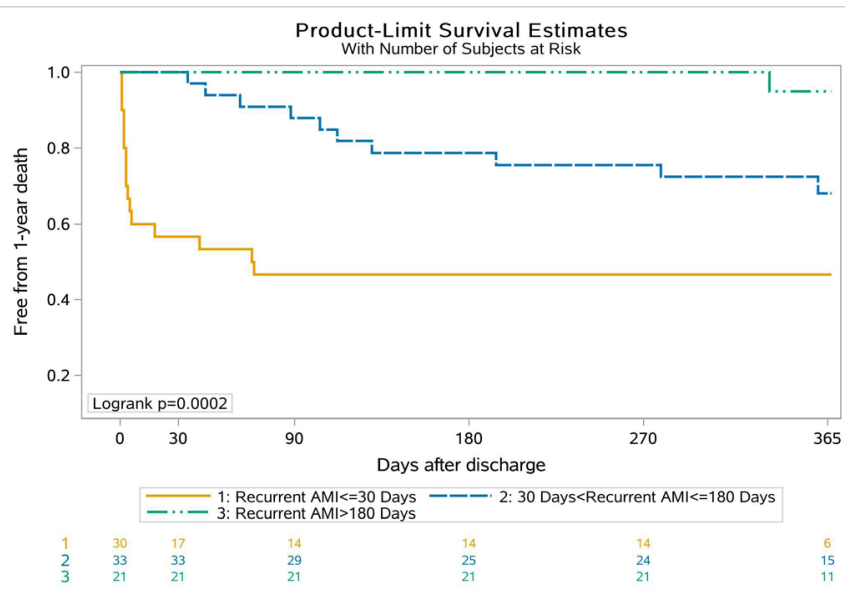

Figure 2 Unadjusted Kaplan-Meier curve for 1-year all-cause death by the timing of recurrent acute myocardial infarction (AMI) relative to discharge.

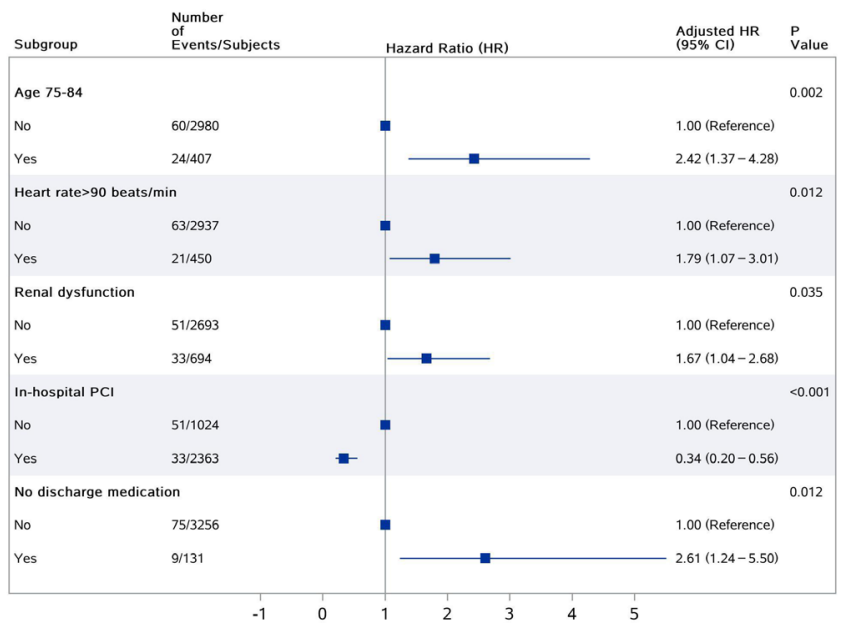

Figure 3 Factors associated with recurrent acute myocardial infarction. $\mathrm{PCl}$, percutaneous coronary intervention.

\section{Factors associated with recurrent AMI}

We identified several factors associated with recurrent AMI in the multivariate model (Figure 3), including advanced age, in-hospital PCI, heart rate $>90 \mathrm{~min} /$ beats at admission, renal dysfunction, and not being prescribed any of the guidelinerecommended medications at discharge.

\section{Discharge therapies}

The composite prescription rate for guideline-recommended medications at discharge was lower among patients with recurrent AMI ( $88.9 \%$ vs $83.2 \%, \mathrm{p}=0.002)$ (Figure 4$)$. Nine hundred and sixty $(28.3 \%)$ patients did not receive all treatments for which they were considered ideal and 98 (10.2\%), 791 (82.4\%), and $71(7.4 \%)$ of these individuals were with high, average, and low risk for 1-year mortality, respectively.

\section{DISCUSSION}

In this real-world cohort of patients with AMI, we found that 1 in 40 patients experienced recurrent AMI within 1 year of discharge, and patients with recurrent AMI had a 25 -fold higher adjusted risk of 1-year mortality. More than one-third of recurrent AMI occurred early (within the first 30 days after discharge), and early recurrent AMI conferred the highest risk for 1-year

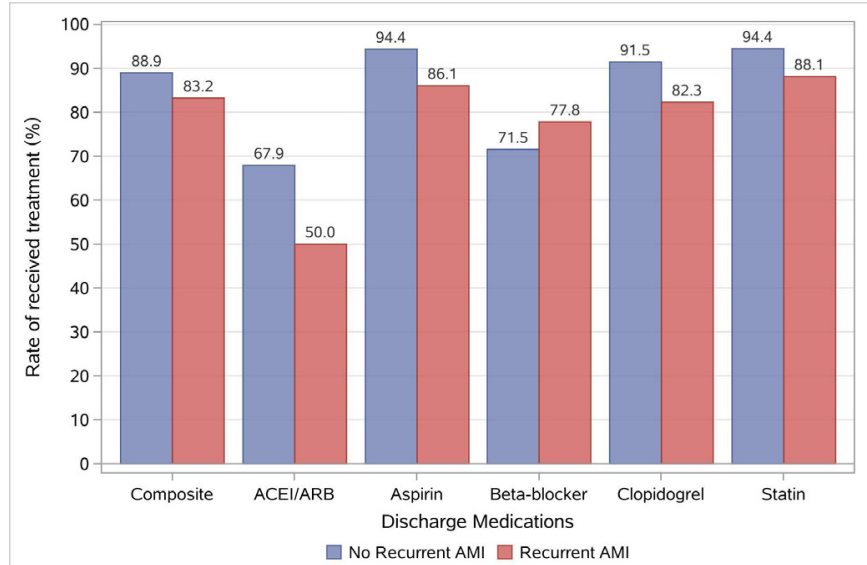

Figure 4 Prescription rates of five guideline-based medications at discharge. AMI, acute myocardial infarction. 
mortality. Inadequate prescription of recommended medications at discharge was associated with recurrent AMI.

We expand on previous literature in several respects. First, our study, based on data from a large real-world cohort of patients followed for 1 year with rigorous adjudication of events, is the first accurate estimate of 1-year postdischarge recurrent AMI rates in China. Rates of recurrent AMI were $2.5 \%$ and $4.1 \%$ among all patients and patients aged $\geq 65$ years, respectively. These findings are similar to a prior report in Japan ${ }^{19}$ and comparable to that observed in Western nations. ${ }^{148}$ Specifically, the HORIZONS-AMI trial enrolling patients with ST-segmentelevation myocardial infarction) reported 1-year recurrent AMI rates of $4.0 \%,{ }^{8}$ while a study among Medicare fee-for-service patients reported 1-year recurrent AMI rates of 5.1\% in 2014. ${ }^{1}$

Second, our study presents an in-depth analysis of the 1-year recurrent AMI-specific pattern after hospitalisation for AMI. Prior studies have focused on readmissions in general $^{20}$ or combined recurrent AMI events with other causes for readmission, ${ }^{21}$ while others have described recurrent AMI patterns for patients specifically after PCI. ${ }^{82}$ We found that a disproportionately high number of recurrent AMI occurred soon after discharge with one-third of events concentrated in the first 30 days, subsequent to which the recurrent AMI rates remain low out to 1 year. These findings reinforce the need for heightened surveillance in this high-risk early phase after AMI discharge. They also imply that hospitals in China should expand their focus from inpatient care alone to transitional care as well. There may be potential for the introduction of hospital performance measures such as 30 -day readmission ${ }^{23}$ to encourage hospitals and physicians to focus on care coordination, early follow-up, and postacute care.

Third, we quantified the impact of recurrent AMI on 1-year mortality and our results suggest a far greater prognostic impact of recurrent AMI on mortality compared with other Western studies. ${ }^{24} 25$ The 1-year mortality was much higher among patients experiencing recurrent AMI in the early period when compared with late recurrent AMI. ${ }^{9}$ The mechanism by which early recurrent AMI is more likely to result in 1-year death than the late is unclear. We observed that patients who experienced early recurrent AMI had higher baseline risk for mortality at the index admission. Thus, it is possible that patients who experience early events are sicker in general. Also, early recurrent AMI events may frequently represent stent thrombosis ${ }^{8}$ which is an event associated with high mortality. Additionally, the early postdischarge phase after any hospital stay regardless of cause has been shown to be a period of increased vulnerability, ${ }^{26}$ with diminished physiological function, depleted reserves and impaired immune function, and events during this time may be associated with excess mortality risk.

Fourth, we identified predictors of recurrent AMI, which is essential for comprehensive risk stratification of patients and recurrent AMI prevention. Prior studies found advanced age, renal insufficiency, and in-hospital PCI to predict recurrent AMI. ${ }^{59}$ Our multivariate regression analysis confirm these predictors but also identify additional predictors, such as increased heart rate at admission and incomplete prescription of recommended medications at discharge. About one in three patients were not prescribed all the guideline-recommended medications for which they were eligible at discharge, and notably, one-tenth of these individuals were at high risk for future death. Although some patients who did not receive all medications for which they were considered ideal had recurrent AMI shortly after discharge, the median time for recurrent AMI was still 42 days postdischarge for these patients. This indicates there was potential to intensify treatment even after discharge and possibly some of the events could have been delayed or prevented with closer follow-up.

Our study results need to be interpreted in the context of certain limitations. First, similar to other longitudinal observational studies, ${ }^{27}$ the study could only include patients who consented to participate. Our findings therefore may not generalise to all patients with AMI. Second, although multivariable analysis was performed to adjust for baseline mortality risk and recurrent AMI was modelled as a time-varying covariate, there might be unmeasured confounders that affect the observed association between recurrent AMI and 1-year mortality. Thus, one cannot directly infer from these analyses that the observed associations establish a causal link between recurrent AMI and mortality. Third, we did not have health information preceding the recurrent AMI event such as triggering events, adherence to secondary prevention medications which limit our understanding of the mechanisms by which these recurrent AMI events occurred. Fourth, we did not have information about the quality of care during the recurrent AMI hospitalisation which has important implications for subsequent outcome. This an important area for future study especially as our results suggest a particularly poor prognosis for recurrent AMI events in China, far greater than that observed in the West. Fifth, in the present study, we only focused on the prescription of five guidelinebased medications recognised as performance measures by the AHA/ACC. Additional research is needed to assess the impact of adherence to other secondary prevention therapies such as aldosterone antagonists.

In conclusion, our analysis from the China PEACE-Prospective AMI study demonstrates that the rate of recurrent AMI within 1 year after discharge is low with more than one-third occurring within the first 30 days after discharge. Recurrent AMI is strongly associated with 1-year mortality, particularly if occurring within the first 30 days. Improvements in prescription of guideline-directed discharge therapies, care coordination and

\section{Key questions}

What is already known on this subject?

- Patients who survive acute myocardial infarction (AMI) in the acute phase remain at risk for recurrent AMI after discharge. Recurrent AMI after hospital discharge is associated with significant morbidity and mortality.

\section{What might this study add?}

- Based on data from a large real-world cohort of patients with AMI in China, we found that 1 in 40 patients experienced recurrent AMI within 1 year after discharge and more than one-third of recurrent AMI occurred early-within the first 30 days after discharge. Recurrent $\mathrm{AMI}$ is strongly associated with 1-year mortality, particularly if occurring early. In addition to clinical factors, inadequate prescription of recommended medications at discharge is associated with recurrent AMI.

\section{How might this impact on clinical practice?}

- Improvements in prescription of guideline-directed discharge therapies, care coordination, and increasing surveillance especially in the 30 days post-AMI may reduce the risk of recurrent AMI. 
increasing surveillance especially in the 30 days post-AMI may reduce the risk of these events.

\section{Author affiliations}

${ }^{1}$ National Clinical Research Center for Cardiovascular Diseases, NHC Key Laboratory of Clinical Research for Cardiovascular Medications, State Key Laboratory of Cardiovascular Disease, Fuwai Hospital, Chinese Academy of Medical Sciences and Peking Union Medical College, National Center for Cardiovascular Diseases, Beijing, China

${ }^{2}$ Section of Cardiovascular Medicine, Department of Internal Medicine, Yale University School of Medicine, New Haven, Connecticut, United States

${ }^{3}$ Yale-New Haven Hospital Center for Outcomes Research and Evaluation, New Haven, Connecticut, United States

${ }^{4}$ Yale School of Public Health, Yale University School of Medicine, and Yale-New Haven Hospital, New Haven, Connecticut, United States

Acknowledgements We appreciate the multiple contributions made by study teams at the National Clinical Research Center for Cardiovascular Diseases and the Yale-New Haven Hospital Center for outcomes research and evaluation in the realms of study design and operation. We thank the support provided by the Chinese government. We thank the patients who participated in this study.

Contributors JS and XZ conceived of this article. JS and KM wrote the manuscript with further contributions from XZ, XL, SH, YG and HMK. JS, SH and YG completed all the statistical analysis. All authors interpreted data, contributed to critical revisions and approved the final version of the article.

Funding This project was supported by the National Key Research and Development Programme (2017YFC1310803) from the Ministry of Science and Technology of China, the CAMS Innovation Fund for Medical Science (2016-I2M-1006), and the 111 Project (B16005) from the Ministry of Education of China.

Competing interests $\mathrm{HMK}$ is the recipient of a research grant from Medtronic and Johnson \& Johnson, through Yale University, to develop methods of clinical trial data sharing; chairs a cardiac scientific advisory board for United Health; works under contract with the Centers for Medicare \& Medicaid Services to develop and maintain performance measures that are publicly reported; is a participant/ participant representative of the IBM Watson Health Life Sciences Board; is a member of the Advisory Board for Element Science and the Physician Advisory Board for Aetna; and is the founder of Hugo, a personal health information platform. HMK and KM work under contract with the Centers for Medicare \& Medicaid Services to develop and maintain performance measures that are publicly reported

Patient and public involvement Patients and/or the public were not involved in the design, or conduct, or reporting, or dissemination plans of this research.

Patient consent for publication Not required.

Ethics approval The central ethics committee at Fuwai Hospital, local ethics committees at participating hospitals and the Yale University Institutional Review Board approved the China Peace-Prospective AMI Study.

Provenance and peer review Not commissioned; externally peer reviewed.

Data availability statement No data are available. It is our future plan to share the data of this study. However, we are unable to do so at this time.

Open access This is an open access article distributed in accordance with the Creative Commons Attribution Non Commercial (CC BY-NC 4.0) license, which permits others to distribute, remix, adapt, build upon this work non-commercially, and license their derivative works on different terms, provided the original work is properly cited, appropriate credit is given, any changes made indicated, and the use is non-commercial. See: http://creativecommons.org/licenses/by-nc/4.0/.

\section{ORCID iD}

Xin Zheng http://orcid.org/0000-0002-2854-8890

\section{REFERENCES}

1 Krumholz HM, Normand S-LT, Wang Y. Twenty-Year trends in outcomes for older adults with acute myocardial infarction in the United States. JAMA Netw Open 2019:2:e191938.

2 Chaudhry SI, Khan RF, Chen J, et al. National trends in recurrent AMI hospitalizations 1 year after acute myocardial infarction in Medicare beneficiaries: 1999-2010. J Am Heart Assoc 2014;3:e001197.

3 Brown TM, Deng L, Becker DJ, et al. Trends in mortality and recurrent coronary heart disease events after an acute myocardial infarction among Medicare beneficiaries, 2001-2009. Am Heart J 2015;170:249-55.
4 Wang Y, Leifheit E, Normand S-LT, et al. Association between subsequent hospitalizations and recurrent acute myocardial infarction within 1 year after acute myocardial infarction. J Am Heart Assoc 2020;9:e014907.

5 Pocock SJ, Mehran R, Clayton TC, et al. Prognostic modeling of individual patient risk and mortality impact of ischemic and hemorrhagic complications: assessment from the acute catheterization and urgent intervention triage strategy trial. Circulation 2010;121:43-51

6 Mehran R, Pocock SJ, Stone GW, et al. Associations of major bleeding and myocardia infarction with the incidence and timing of mortality in patients presenting with nonST-elevation acute coronary syndromes: a risk model from the acuity trial. Eur Heart J 2009;30:1457-66.

7 Lewis EF, Li Y, Pfeffer MA, et al. Impact of cardiovascular events on change in quality of life and utilities in patients after myocardial infarction: a VALIANT study (valsartan in acute myocardial infarction). JACC Heart Fail 2014;2:159-65.

8 Stone SG, Serrao GW, Mehran R, et al. Incidence, predictors, and implications of reinfarction after primary percutaneous coronary intervention in ST-segment-elevation myocardial infarction: the Harmonizing outcomes with revascularization and stents in acute myocardial infarction trial. Circ Cardiovasc Interv 2014;7:543-51.

9 Thune JJ, Signorovitch JE, Kober L, et al. Predictors and prognostic impact of recurrent myocardial infarction in patients with left ventricular dysfunction, heart failure, or both following a first myocardial infarction. Eur J Heart Fail 2011;13:148-53.

10 Dharmarajan K, Hsieh AF, Lin Z, et al. Diagnoses and timing of 30-day readmissions after hospitalization for heart failure, acute myocardial infarction, or pneumonia. JAMA 2013;309:355-63.

11 Kim LK, Yeo I, Cheung JW, et al. Thirty-Day readmission rates, timing, causes, and costs after ST-segment-elevation myocardial infarction in the United States: a national readmission database analysis 2010-2014. J Am Heart Assoc 2018:7:e009863.

12 Wang S, Marquez P, Langenbrunner J, et al. Toward a healthy and harmonious life in China: stemming the rising tide of non-communicable diseases. Washington: The World Bank, 2011.

13 Li J, Dreyer RP, Li X, et al. China patient-centered Evaluative assessment of cardiac events prospective study of acute myocardial infarction: study design. Chin Med J 2016;129:72-80

14 Thygesen K, Alpert JS, Jaffe AS, et al. Third universal definition of myocardial infarction. J Am Coll Cardiol 2012;60:1581-98.

15 Wang $Y$, Li J, Zheng $X$, et al. Risk factors associated with major cardiovascular events 1 year after acute myocardial infarction. JAMA Netw Open 2018;1:e181079.

16 Ineid $\mathrm{H}$, Addison D, Bhatt DL, et al. 2017 AHA/ACC clinical performance and quality measures for adults with ST-elevation and Non-ST-Elevation myocardial infarction. $J$ Am Coll Cardiol 2017;70:2048-90.

17 HPS2-THRIVE Collaborative Group. HPS2-THRIVE randomized placebo-controlled trial in 25673 high-risk patients of ER niacin/laropiprant: trial design, pre-specified muscle and liver outcomes, and reasons for stopping study treatment. Eur Heart $J$ 2013;34:1279-91.

18 Sterne JAC, White IR, Carlin JB, et al. Multiple imputation for missing data in epidemiological and clinical research: potential and pitfalls. BMJ 2009;338:b2393.

19 Nakatani D, Sakata Y, Suna S, et al. Incidence, predictors, and subsequent mortality risk of recurrent myocardial infarction in patients following discharge for acute myocardial infarction. Circ J 2013;77:439-46.

20 Dreyer RP, Dharmarajan K, Kennedy KF, et al. Sex differences in 1-year all-cause rehospitalization in patients after acute myocardial infarction: a prospective observational study. Circulation 2017:135:521-31.

21 Khot UN, Johnson MJ, Wiggins NB, et al. Long-Term time-varying risk of readmission after acute myocardial infarction. J Am Heart Assoc 2018:7:e009650.

22 Vink MA, Vos NS, Dirksen MT, et al. Recurrent myocardial infarction after primary percutaneous coronary intervention in multivessel coronary disease is primarily related to stent failure: Post-Hoc analysis of the passion trial. J Interv Cardiol 2015;28:523-30.

23 Krumholz HM, Lin Z, Drye EE, et al. An administrative claims measure suitable for profiling Hospital performance based on 30-day all-cause readmission rates among patients with acute myocardial infarction. Circ Cardiovasc Qual Outcomes 2011;4:243-52.

24 Stone GW, Clayton T, Deliargyris EN, et al. Reduction in cardiac mortality with bivalirudin in patients with and without major bleeding: the HORIZONS-AMI trial (Harmonizing outcomes with revascularization and stents in acute myocardial infarction). J Am Coll Cardiol 2014;63:15-20.

25 Kikkert WJ, Zwinderman AH, Vis MM, et al. Timing of mortality after severe bleeding and recurrent myocardial infarction in patients with ST-segment-elevation myocardial infarction. Circ Cardiovasc Interv 2013;6:391-8.

26 Krumholz HM. Post-hospital syndrome--an acquired, transient condition of generalized risk. N Engl J Med 2013;368:100-2.

27 Arnold SV, Chan PS, Jones PG, et al. Translational research investigating underlying disparities in acute myocardial infarction patients' health status (triumph): design and rationale of a prospective multicenter registry. Circ Cardiovasc Qual Outcomes 2011;4:467-76. 